\title{
The impact of teamwork on missed nursing care
}

\author{
Beatrice J. Kalisch, RN, PhD, FAAN \\ Kyung Hee Lee, RN, MPH
}

\begin{abstract}
Previous studies have shown that missed nursing care is a significant problem in acute care hospitals. Other studies have demonstrated that teamwork is a critical element in assuring patient safety and quality of care. The purpose of this study was to determine if the level of nursing teamwork impacts the extent and nature of missed nursing care. A sample of 2216 nursing staff members on 50 acute care patient care units in 4 hospitals completed the Nursing Teamwork Survey and the MISSCARE Survey. The response rate was $59.7 \%$. Controlling for occupation of staff members (eg, RN/LPN, NA) and staff characteristics (eg, education, shift worked, experience, etc), teamwork alone accounted for about $11 \%$ of missed nursing care. The results of this study show that the level of nursing teamwork impacts the nature and extent of missed nursing care. The study results point to a need to invest in methods of enhancing teamwork in these settings.
\end{abstract}

I thas been recognized that teamwork among healthcare providers is essential for patient safety. The Institute 1 of Medicine (IOM) study, To Err is Human, for example, was one of the first studies which pointed to the need for enhanced teamwork in health care to avoid patient errors. ${ }^{1}$ The follow-up report by IOM also clearly indicated that nurses are indispensable to patient safety. ${ }^{2}$ Despite the rising interest in teamwork, most studies of teamwork in health care have focused on emergency and perioperative settings. There has been very little attention given to nursing teamwork on inpatient units in acute care hospitals. Given the fact that there are literally thousands of such teams providing nursing care to patients throughout the world, the potential impact on patient safety and quality of care is enormous.

Beatrice J. Kalisch, RN, PhD, FAAN, is Titus Distinguished Professor of Nursing and Director, Nursing Business and Health Systems, University of Michigan, School of Nursing, Ann Arbor, MI.

Kyung Hee Lee, RN, MPH, is a Research Assistant and PhD Candidate at University of Michigan, School of Nursing, Ann Arbor, MI.

Corresponding Author: Dr. Beatrice J. Kalisch, University of Michigan, School of Nursing, 400 N. Ingalls Street, Ann Arbor, MI 48109.

E-mail: bkalisch@umich.edu

Nurs Outlook 2010;58:233-241.

0029-6554/\$ - see front matter

(c) 2010 Mosby, Inc. All rights reserved.

doi:10.1016/j.outlook.2010.06.004
The following are examples of teamwork problems leading to a poor quality of nursing care or specifically missed nursing care uncovered in a qualitative study of nursing teamwork ${ }^{3}$ :

- A nursing assistant (NA) sees a medication (Glucophage-oral insulin) left in a cup on a patient's bed stand and throws it way, never telling the Registered Nurse (RN). The patient's blood sugar is elevated on the next blood test and the RN cannot understand why. The dose of Glucophage is increased.

- A nurse requests help to ambulate a $300 \mathrm{lb}$ patient from another nurse who says she will come but, 2 hours later, she has not shown up. The nurse then tries to get the patient up by herself. The patient falls and is injured and the nurse has to take a medical leave for a back problem.

- A nurse assistant says she does not understand the need for the nurse to document so much of the time. She interprets this as "not putting the patient first" and decides to not answer call lights promptly because why should she do it if the RN is not doing it herself?

- A patient is brought up to the unit from the emergency department by a transporter and informs an RN \#1. RN \#1 fails to notify RN \#2 assigned to this patient and 2 hours pass before $\mathrm{RN} \# 2$ assigned to the patient discovers that the patient has arrived on the unit. In the meantime, the patient is in intense pain. RN \#2 does not address this with the other RN. A month later the same RN does the same thing with another patient brought to the unit and the patient is dead when the $\mathrm{RN}$ assigned to the patient finally gets into the room.

The purpose of this study is to determine if the presence or absence of nursing teamwork results in missed nursing care, which is any aspect of required patient care that is omitted or significantly delayed. ${ }^{4}$ As such, missed nursing care is an indicator of the quality of nursing care.

\section{BACKGROUND}

Missed Nursing Care

A detailed literature review suggests a lack of clarity regarding the degree to which nursing care is provided or not. Findings from completed studies, largely observational, have revealed aspects of nursing care not completed-namely ambulation, turning, nutrition, 
medications, patient teaching, hand washing, and intravenous site care..$^{5-13}$

Ambulation standards for hospitalized patients indicate that patients should be progressively mobilized 3 times per day. ${ }^{13}$ However, the frequency of hallway walking by adults hospitalized on a medical unit was found to be as follows: only $19 \%$ walked once, $5 \%$ twice, $3 \%$ more than twice, and $73 \%$ did not walk at all. $^{7}$ Although an accepted standard of care is turning immobilized patients in bed every 2 hours, researchers found in an observational study of 74 intensive care patients for 566 hours that only 2 of the 74 patients $(2.7 \%)$ had a change in body position every 2 hours. ${ }^{7}$

One study uncovered the fact that $40 \%$ of the hospitalized patients were malnourished upon admission and that $75 \%$ lost further weight during hospitalization. ${ }^{10}$ Omission of ordered medications has been reported to be the most common medication error. ${ }^{5}$ In a survey of patients discharged from medical-surgical units, almost half stated they needed additional information or specific directions concerning their self-care. ${ }^{9}$ The rate of handwashing has been discovered to be at the $40 \%$ level. ${ }^{11,12}$ Looking at missed intravenous site care, 1 study revealed that $72 \%$ changed the peripheral locks only when necessary while $6.2 \%$ of respondents indicated they completed this task every 5 days and another $3.1 \%$ every 48 hours. $^{6}$

The overall concept of missed nursing care was first reported in $2006 .{ }^{14}$ Missed nursing care are errors of omission (eg, not teaching the patient) as opposed to errors of commission (eg, giving the incorrect medication). ${ }^{14} \mathrm{In}$ addition, 2 related concepts have been investigated - unfinished care ${ }^{15}$ and rationed care. ${ }^{16}$ Sochalski showed that the quality of nursing care was strongly related to rates of unfinished care for those patients $(r=.634$, $P<.001) .{ }^{15}$ In addition, a team in Switzerland investigated rationed nursing care and found that although nursing staff reported a low level of care not completed, it was a predictor of 6 patient outcomes (ie, patient satisfaction, nurse-reported medication errors, patient falls, nosocomial infections, pressure ulcers, and critical incidents involving patients over the previous year). ${ }^{16}$

A qualitative study of missed nursing care identified 9 areas of missed care (ambulation, turning, delayed or missed feedings, patient teaching, discharge planning, emotional support, hygiene, intake and output documentation and surveillance) and 7 reasons for missing that care (eg, too few staff time required for the nursing intervention, poor teamwork, etc). ${ }^{14}$

Since there was no available instrument to measure missed nursing care quantitatively, a tool-The MISSCARE Survey-was developed and tested for its psychometric properties. ${ }^{17}$ Using this instrument, a 3-hospital study of nurses $(\mathrm{N}=459)$ uncovered a substantial amount of missed care (mean score is 1.85 with a range of 1.00-3.48). The 6 most frequently missed care activities were ambulation (84\%), assessing of the effec- tiveness of medications (83\%), turning (82\%), mouth care $(82 \%)$, patient teaching $(80 \%)$, and the timely administration of PRN (as needed) medications (80\%).

Another study provided data as to the extent, type of, and reasons for missed nursing care and variations of missed nursing care in 10 hospitals on 110 patient care units $(\mathrm{N}=4288$ ) (Kalisch B, Tschannen D, Hyunhwa L, Friese CR. Hospital variation in missed nursing care, unpublished data). Although there were pairs of hospitals that were significantly different in the amount of missed care (eg, hospital 2 had significantly higher rates of not turning patients than hospital 7), the most missed and least missed elements of care were similar across the 10 hospitals. Elements of care missed the most across all 10 hospitals were ambulation (76\%), interdisciplinary care conference attendance $(65 \%)$, mouth care $(64 \%)$, timely medication administration $(60 \%)$, and turning (58\%) while those missed the least were assessment each shift (10\%), glucose monitoring (14\%), patient discharge planning and teaching $(25 \%)$, vital signs (25\%), and focused reassessment (27\%).

\section{Teamwork}

Research on teamwork across industries is extensive but, within healthcare and nursing, is much more limited. Most studies in healthcare have focused on interdisciplinary teamwork. For example, a study of intensive care staff showed that interdisciplinary teams reporting a higher level of team development had lower patient mortality rates. ${ }^{18}$ Brewer found that a "group type hospital culture" resulted in fewer patient falls, ${ }^{19}$ and Morey and colleagues discovered that higher teamwork led to fewer errors. ${ }^{20}$ An intervention to improve teamwork resulted in significant improvement in microalbumin testing of diabetic patients. ${ }^{21}$ Another research team found a significant positive relationship between measures of hospital teamwork culture and patient satisfaction. ${ }^{22}$

Looking specifically at teamwork within nursing (among nurses and assistive personnel) working on a given acute care patient unit, as opposed to healthcare staff members who work on several (or all) patient units (eg, physicians, physical therapists, pharmacists, etc), we uncovered scant research. Several investigators have reported a link between nursing staff satisfaction and teamwork, ${ }^{23-26}$ and one study linked higher levels of nursing teamwork with fewer patient falls, lower vacancy, and turnover rates. ${ }^{27}$ No studies of the impact of teamwork on the process of nursing care were found.

\section{CONCEPTUAL FRAMEWORK}

The conceptual framework for this study is illustrated in Figure 1. This conceptual framework postulates that organizational and patient unit characteristics, along with the level of nursing teamwork, predict missed nursing care. The individual staff member demographic characteristics (ie, gender, age, education, and experience) 


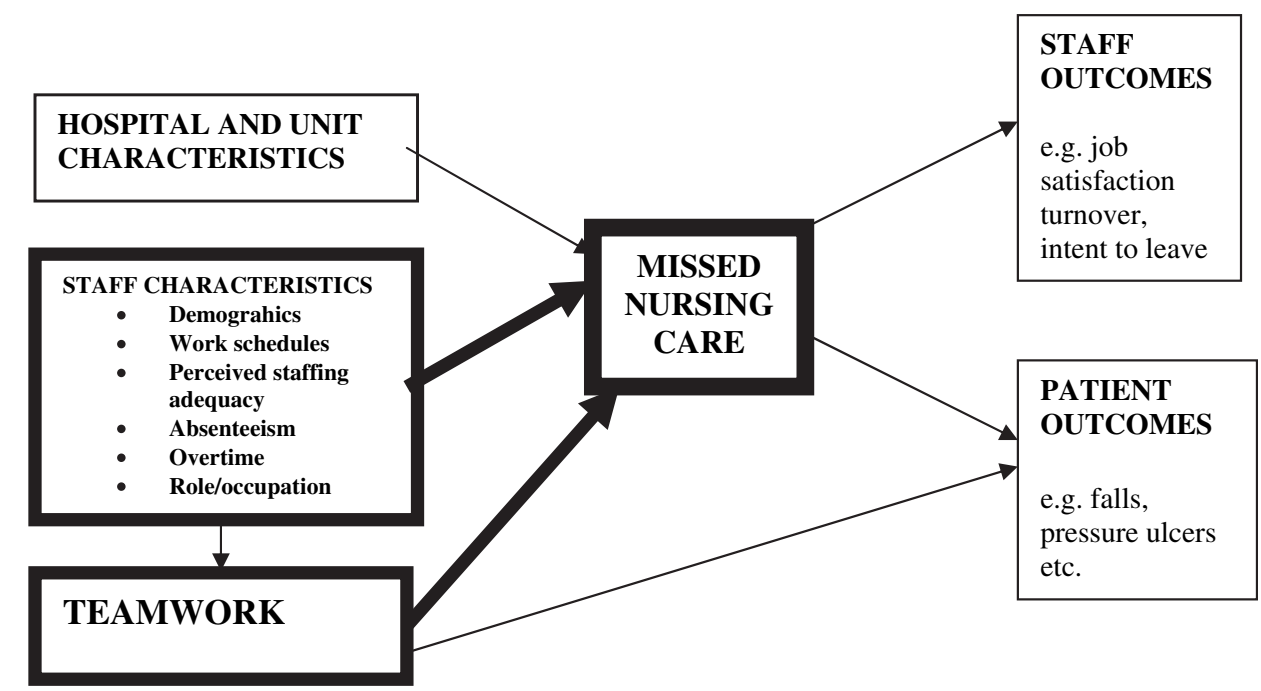

Figure 1. The Missed Nursing Care Model (current study highlighted)

along with their occupation or role (ie, $\mathrm{RN}$, licensed practical nurse [LPN], NA), work schedules (full or part-time, shift worked), staffing adequacy, overtime, and absenteeism are hypothesized to potentially contribute to variances in missed care and thus need to be controlled in the model. For example, previous studies have shown that experience, length of shift worked, shift (days, nights), number of hours worked per week, perceptions of staffing adequacy, extent of overtime and absenteeism influenced teamwork scores. ${ }^{28}$ Variances by occupation (RN, LPN, NA) have also been found for both missed nursing care and nursing teamwork. ${ }^{28,29}$

While over 130 theories of teamwork have been developed, ${ }^{30}$ Salas, Sims and Burke's conceptual framework of teamwork was selected to use in this study because it offers a practical, measureable, and easy-tounderstand explanation of teamwork. ${ }^{31}$ They identify 5 core components and 3 coordinating functions. The core components are: (1) team leadership (ie, structure, direction and support provided by both the formal leader and/or by other members of the team), (2) team orientation (ie, individuals see the team's success as more important than the individual's achievements), (3) mutual performance monitoring (ie, observation and awareness of team members), (4) backup behavior (ie, assisting other team members when they need help), and (5) adaptability (ie, ability to alter approach based on changes in the environment). The 3 coordinating mechanisms are: (1) shared mental models (ie, mutual conceptualizations of what needs to be done, who should do it, and what processes and strategies are needed to complete the work), (2) closed-loop communication (ie, active information exchange where the receiver acknowledges receipt and the sender verifies intended message was received), and (3) mutual trust (ie, shared perception that members will perform actions necessary to reach interdependent goals and act in the interest of the team). ${ }^{31}$ This framework has been applied to inpa- tient nursing teams and results support the framework as a means for describing teamwork among nurses in these settings. ${ }^{31}$

\section{RESEARCH QUESTIONS}

The aim of this study was to examine the effect of nursing teamwork on missed nursing care. The research questions are as follows:

How does the level of team work vary by unit type?

- Does nursing teamwork predict missed nursing care after controlling for occupation of staff members (eg, RN/ LPN, NA), and staff characteristics (education, gender, age, experience, absenteeism, work schedules, and perceptions of staffing adequacy)?

\section{METHOD}

\section{Sample and Setting}

The setting for this cross-sectional, descriptive study was 50 medical-surgical, intermediate, intensive care, and rehabilitation units in 4 hospitals located in the Midwest. As seen in Table 1, the sample was made up of 2216 nursing staff members (1 719 staff nurses (RNs/LPNs) and 491 NAs). Licenses Practical Nurses accounted for only $1.3 \%(n=29)$ of the sample and thus was too small for comparison purposes. Therefore, they were combined into a category for all nurses. Among a total of 2216 participants, $89.2 \%$ were female, $34.1 \%$ had $>10$ years of experience in their role, $23.7 \%$ had 6 months to 2 years of experience in their role, $42.6 \%$ held a baccalaureate degree, $45.0 \%$ worked the day shift, $84.3 \%$ worked for $\geq 30$ hours weekly, and $51.3 \%$ worked on medical-surgical units (Table 1).

\section{Study Instruments}

Two survey instruments were utilized in this studythe MISSCARE Survey and the Nursing Teamwork 


\section{Table 1. Characteristics of the study sample $(N=2216)$}

\begin{tabular}{|c|c|c|c|}
\hline Variable & Category & Frequency & Percent \\
\hline \multirow[t]{2}{*}{ Gender } & Male & 233 & 10.8 \\
\hline & Female & 1929 & 89.2 \\
\hline \multirow[t]{5}{*}{ Age } & $\sim 25 \mathrm{yrs}$ old & 323 & 14.7 \\
\hline & $26 \sim 34$ yrs old & 633 & 28.7 \\
\hline & $35-44$ yrs old & 589 & 26.7 \\
\hline & $45-54$ yrs old & 463 & 21.0 \\
\hline & $55+$ yrs old & 196 & 8.9 \\
\hline \multirow{5}{*}{ Yrs of experience in the role } & $\sim 6 \mathrm{mths}$ & 112 & 5.1 \\
\hline & Greater than $6 m$ ths to $2 y r s$ & 521 & 23.7 \\
\hline & Greater than 2yrs to 5yrs & 424 & 19.3 \\
\hline & Greater than 5yrs to 10yrs & 389 & 17.7 \\
\hline & Greater than 10yrs & 749 & 34.1 \\
\hline \multirow[t]{4}{*}{ Highest education } & Grade, High school grad & 323 & 14.7 \\
\hline & Associate & 844 & 38.5 \\
\hline & Bachelor & 935 & 42.6 \\
\hline & Graduate school & 92 & 4.2 \\
\hline \multirow{2}{*}{ Job } & Staff Nurse (RN /LPN) & 1719 & 77.8 \\
\hline & Nursing Assistant & 491 & 22.2 \\
\hline \multirow[t]{4}{*}{ Shift worked } & Day & 995 & 45.0 \\
\hline & Evening & 218 & 9.9 \\
\hline & Night & 725 & 32.8 \\
\hline & Rotating & 273 & 12.3 \\
\hline \multirow{2}{*}{ Employment status } & > 30 hrs/week & 1866 & 84.3 \\
\hline & $\leq 30 \mathrm{hrs} /$ week & 348 & 15.7 \\
\hline \multirow[t]{4}{*}{ Unit type } & Intensive care & 631 & 28.5 \\
\hline & Intermediate & 357 & 16.1 \\
\hline & Medical-surgical & 1136 & 51.3 \\
\hline & Rehabilitation & 91 & 4.1 \\
\hline
\end{tabular}

Survey (NTS). The psychometric properties of each tool have been published elsewhere. ${ }^{32,33}$ A summary of these results are presented below.

Nursing Teamwork Survey. The Nursing Teamwork Survey (NTS) was designed to specifically measure nursing teamwork in inpatient settings. The NTS is a 33-item questionnaire with a Likert-type scaling system $(1=$ rarely to $5=$ always). Validity was tested first with expert panels who achieved a content validity index (CVI) of 0.91, indicating high consistency among ratings of item relevance and clarity. Construct validity, tested with exploratory factor analysis (EFA), resulted in a 5-factor solution: trust (7 items), team orientation (9 items), backup (6 items), shared mental models ( 7 items) and team leadership (4 items). These 5 factors with 33 items in total explained $53.11 \%$ of the variance. The result from the Bartlett Test indicated that the correlation matrix is not an identity matrix $\left(x^{2}=12860.195, \mathrm{df}=528, P<.01\right)$, and the KMO measure showed that sampling adequacy was excellent (0.961).

Confirmatory Factor Analysis (CFA) demonstrated that the 5-factor model fits the data well. A Comparative
Fit Index (CFI) of the model was 0.884, the Root Mean Square Error of Approximation (RMSEA) index was 0.055 , and the Standardized Root Mean Residual (SRMR) fit index was 0.045 . Convergent validity involved a comparison of scores on the Safety Attitudes Questionnaire subscale on teamwork. ${ }^{34}$ The NTS was correlated $(\mathrm{r}=0.76, P<.01)$ to this subscale. ${ }^{32}$ Testretest reliability was 0.92 and the coefficients on each subscale ranged from $0.77-0.87$. The overall internal consistency of the survey was 0.94 , and the $\alpha$ coefficients on each subscale ranged from $0.74-0.85$. Intra-class correlation coefficients (ICC) and index of agreement $\left(r_{w g(j)}\right)$ were also calculated to estimate interrater agreement for each unit staff group. These analyses demonstrated members of the same unit responded similarly to the NTS and staff from different units responded differently.

MISSCARE Survey. The MISSCARE Survey A is a 24-item survey instrument which asks nursing staff to identify how frequently the elements of nursing care (such as ambulation, turning, patient assessment, teaching, discharge planning, medication administration, etc.) are missed, using a Likert scale, with anchors "rarely 
missed" to "always missed." The items on the survey were based on a focus group study, ${ }^{14}$ other staff interviews, and the literature. Panels of experts were employed to review the instrument for face/content validity. Pearson correlation coefficient on a test-retest of the same subjects yielded a 0.87 .

Staff characteristics. Gender, age, education, experience, and occupation (ie, RN/ LPN, NA), work schedules (full or part-time, shift worked), perceptions of staff adequacy, overtime, and absenteeism were also collected from each study participant. Staffing adequacy was assessed with the question, "How often do you feel the unit staffing is adequate?" They were given 5 choices: $0 \%, 25 \%, 50 \%, 75 \%$ or $100 \%$ of the time. The number of hours of overtime in the past 3 months was utilized for this measure while perceived absenteeism was measured using the question, "In the past 3 months, how many days (shifts) did you miss work (exclusive of approved time off)? Validity was tested for these questions with expert panels who achieved a CVI of 0.96, indicating high consistency among ratings of item relevance and clarity. A group of 30 staff members were administered these questions with a 2week or greater gap in time. The resultant test-retest correlations ranged from $0.78-0.95$.

\section{Procedures}

After Internal Review Board (IRB) approval at each facility and orientation of the nursing staff and unit managers to the study, data collection was conducted over a 4-week period of time in each hospital in 2009. The 2 surveys (NTS and the MISSCARE Survey) were combined into one document, placed in a large envelope with a cover letter explaining the study, informing the participants of the confidentiality of their responses and that their participation was voluntary. Surveys were placed in staff mail boxes. Also included was a letter-size envelope in which the participants were instructed to place their completed questionnaires, seal it and place it in a locked box on each patient care unit. For units who achieved a 50\% return rate, pizza was provided. Return rate in this study was $59.7 \%$.

\section{Data Analysis}

The Statistical Package for the Social Science (SPSS), version 17.0 (SPSS, Chicago), and Stata 10 SE were used for data analyses. Stata was employed for the multiple regression analysis with the cluster command because the SPSS cannot perform the cluster option. The level of analysis for this study was the individual nursing staff member. For the missed care variable, the overall mean score of missed care, which was the average amount of missed care identified for each of the elements of nursing care for each participant, was used. The overall mean score of teamwork was also used for the overall teamwork variable.

Preliminary analyses of the data were completed using descriptive and bivariate analysis techniques. Either $t$-test or Analysis of Variance (ANOVA) was used to examine differences in missed care scores by staff characteristics. Pearson correlation analysis was employed to address the relationship between teamwork and missed nursing care. Multiple regression analyses were performed to determine the predictive ability of the overall teamwork score and all significant staff characteristics variables $(P<.05)$ on the dependent variable, missed nursing care. Multicollinearity was examined using the variance inflation factor (VIF).

Due to potential issues with clustering of data by nursing unit, ICC which quantitatively measure the strength of correlation of each unit member response to the group, were estimated by using 1-way ANOVA. Responses within each unit were significantly similar for the missed care variable $\left(F\left[\begin{array}{ll}51 & 2277\end{array}\right]=15.670\right.$, $P<.000$, ICC $=0.21)$. Based on this ICC, missed care scores for the same patient care unit were correlated. To account for data clustering, multiple linear regression analysis was performed with the cluster command in the Stata 10 program to determine relationships between missed care, teamwork, and staff characteristics. In addition, accounting for hospital effect (ie, nesting of data), the hospital variable was included as a predictor.

\section{FINDINGS}

\section{Teamwork by Unit Type}

Table 2 shows teamwork overall score was significantly different by type of unit $(\mathrm{F}=29.14, P<.01)$.

\section{Table 2. Teamwork overall score by unit type}

\begin{tabular}{|llllll}
\hline Variable & \multicolumn{1}{c}{ Categories } & N & Mean \pm SD & F & P \\
\hline Unit & a) Intensive care & 628 & $3.68 \pm .53^{\text {abc }}$ & 29.14 & .00 \\
& b) Intermediate & 355 & $3.49 \pm .62^{\mathrm{a}}$ & & \\
& c) Medical-surgical & 1131 & $3.42 \pm .61^{\mathrm{b}}$ & & \\
& d) Rehabilitation & 91 & $3.37 \pm .57^{\mathrm{c}}$ & & \\
\hline
\end{tabular}

Note: ${ }^{a-c}$ Groups with same letter are significantly different according to Bonferroni post hoc test. 
Post-hoc analysis revealed that intensive care units had a higher teamwork overall score than intermediate, medical-surgical, and rehabilitation units.

\section{Teamwork and Missed Care}

Table 3 presents Pearson correlations for all missed care and teamwork variables. The negative relationship between the missed care mean scores and teamwork overall scores $(\mathrm{r}=-.37, P<.01)$ was supported. More missed care was also significantly related to the following factors: trust $(\mathrm{r}=-.31, P<.01)$, team orientation $(\mathrm{r}=-.28, P<.01)$, backup $(\mathrm{r}=-.31, P<.01)$, shared mental model $(\mathrm{r}=-.32, P<.01)$, and team leadership $(\mathrm{r}=-.29, P<.01)$. The higher the overall teamwork and the subscales scores, the less care was missed.

\section{Predictors of Missed Care}

Based on the preliminary bivariate analyses, age $(\mathrm{F}=1.964, P=.097)$, full-time $(t=-.349$, $P=.555)$, unit type $(\mathrm{F}=.412, P=.744)$ and overtime $(\mathrm{F}=1.444, P=.236)$ were excluded from further analyses because missed nursing care was not significantly associated with these variables. The following variables were included in multivariate analyses to determine predictors of missed nursing care: teamwork overall scores, education, gender, occupation, shift worked, years of experience in the occupation, absenteeism, and perceived adequacy of staffing. The subscales of teamwork were dropped from the model because the teamwork overall scores and the subscales were highly correlated.

In order to evaluate partial $r$-square for the teamwork overall score, 2 models are presented (Table 4). With significant staff characteristics, Model 1 accounted for $11.8 \%$ of the variation in missed nursing care $(\mathrm{F}[18$, $49]=31.14, P<.001)$. After adding the teamwork overall score to the independent variable group, the overall model (Model 2) accounted for $22.7 \%$ of the variation in missed nursing care $(\mathrm{F}[19,49]=54.83, P<.001)$.
Therefore, teamwork overall score alone accounted for about $11 \%$ of the missed nursing care. The effect size in$\operatorname{dex}\left(f^{2}\right)$ of teamwork is .124 , which is close to a medium effect size. ${ }^{35}$ Nurse Assistants perceived less missed nursing care than nurses $(\mathrm{B}=-.184, P=.010)$. Comparing staff with $\leq 6$ months experience, those with 5-10 years of experience $(\mathrm{B}=.084, P=.018)$ and those with $>10$ years experience perceived more missed nursing care $(\mathrm{B}=.089, P=.003)$. Respondents who perceived their unit staffing level to be high reported less missed nursing care than staff who felt staffing was inadequate $(\mathrm{B}=.080, P<.001, \mathrm{~B}=.103, P=.003$ respectively). Missed nursing care was not significantly different across the hospitals (output was suppressed for confidentiality). The VIF of Model 2 ranged from 1.05-5.23.

\section{DISCUSSION}

The results of this study show that the level of nursing teamwork on inpatient acute care hospital patient units impacts the process of nursing care. The process of nursing care in this study was measured by the nursing staff reports of care they did not complete (missed nursing care). When teamwork was stronger, less missed nursing care was reported. This relationship persists after adjustment for individual characteristics of subjects and clustering of data by nursing units and hospitals.

The importance of teamwork among nurse providers in inpatient care units has been little understood and largely ignored. This study provides evidence that teamwork is critical for the provision of quality and safe nursing care. In practice, the demands for patient care vary from moment to moment. For example, if there are numerous admissions at once and/or a patient needs immediate attention, the nurse must prioritize what he or she is going to do first, second, etc. In addition, the environment in which nurses work is characterized by numerous interruptions and multitasking. ${ }^{36}$ Inevitably, the nurse will not be able to provide all of the required care by herself. When teamwork is present, it is much more likely

\section{Table 3. Missed Care and Teamwork: Correlation Matrix}

\begin{tabular}{|c|c|c|c|c|c|c|c|}
\hline Variables & 1 & 2 & 3 & 4 & 5 & 6 & 7 \\
\hline $\begin{array}{l}\text { 1. Missed care } \\
\text { 2. Teamwork overall } \\
\text { 3. Trust } \\
\text { 4. Team orientation } \\
\text { 5. Backup } \\
\text { 6. Shared mental model } \\
\text { 7. Team leadership }\end{array}$ & $\begin{array}{l}- \\
-.37^{\dagger} \\
-.31^{\dagger} \\
-.28^{\dagger} \\
-.31^{\dagger} \\
-.32^{\dagger} \\
-.29^{\dagger}\end{array}$ & $\begin{array}{c}- \\
.87^{\dagger} \\
.74^{\dagger} \\
.83^{\dagger} \\
.84^{\dagger} \\
.75^{\dagger}\end{array}$ & $\begin{array}{c}- \\
.45^{\dagger} \\
.75^{\dagger} \\
.76^{\dagger} \\
.64^{\dagger}\end{array}$ & $\begin{array}{c}- \\
.40^{\dagger} \\
.46^{\dagger} \\
.34^{\dagger}\end{array}$ & $\begin{array}{c}- \\
.69^{\dagger} \\
.69^{\dagger}\end{array}$ & $\frac{-}{.62^{\dagger}}$ & - \\
\hline \multicolumn{8}{|l|}{ * $P<.05$} \\
\hline$\dagger P<.01$ & & & & & & & \\
\hline
\end{tabular}




\begin{tabular}{|c|c|c|c|c|c|c|c|c|}
\hline \multirow[b]{2}{*}{ Variable } & \multicolumn{4}{|c|}{ Model 1} & \multicolumn{4}{|c|}{ Model 2} \\
\hline & B & RobustSE & $t$ & $P$ & B & RobustSE & $t$ & $P$ \\
\hline \multicolumn{9}{|l|}{ Education } \\
\hline \multicolumn{9}{|l|}{$\begin{array}{l}\text { Grade, high } \\
\text { school }(R)\end{array}$} \\
\hline Associate & .009 & .033 & 0.27 & 0.790 & .006 & .032 & 0.19 & 0.846 \\
\hline Baccalaureate & .033 & .044 & 0.76 & 0.449 & .036 & .042 & 0.86 & 0.393 \\
\hline Graduate school & .109 & .069 & 1.58 & 0.120 & .087 & .067 & 1.30 & 0.201 \\
\hline \multicolumn{9}{|l|}{ Gender } \\
\hline \multicolumn{9}{|l|}{ Female $(R)$} \\
\hline Male & -.037 & .026 & -1.42 & 0.162 & -.021 & .027 & -0.77 & 0.442 \\
\hline \multicolumn{9}{|l|}{ Occupation } \\
\hline \multicolumn{9}{|l|}{ Staff nurse (RN/LPN) (R) } \\
\hline Nursing assistant & -.166 & .070 & -2.38 & 0.021 & -.184 & .069 & -2.68 & 0.010 \\
\hline \multicolumn{9}{|l|}{ Shift worked } \\
\hline \multicolumn{9}{|l|}{ Day $(R)$} \\
\hline Evening & -.027 & .030 & -0.91 & 0.369 & -.017 & .029 & -0.57 & 0.571 \\
\hline Night & -.084 & .029 & -2.90 & 0.006 & -.053 & .034 & -1.56 & 0.125 \\
\hline Rotating & -.048 & .031 & -1.53 & 0.133 & -.040 & .031 & -1.28 & 0.208 \\
\hline \multicolumn{9}{|l|}{$\begin{array}{l}\text { Yrs of experience } \\
\text { in the occupation } \\
\sim 6 m t h s(R)\end{array}$} \\
\hline$>6 m$ ths-2yrs & .074 & .046 & 1.61 & 0.113 & .026 & .041 & 0.64 & 0.528 \\
\hline$>2-5 y r s$ & .113 & .044 & 2.55 & 0.014 & .052 & .043 & 1.21 & 0.233 \\
\hline$>5-10 y r s$ & .159 & .031 & 5.19 & 0.000 & .084 & .034 & 2.45 & 0.018 \\
\hline$>10 y r s$ & .163 & .031 & 5.26 & 0.000 & .089 & .029 & 3.12 & 0.003 \\
\hline \multicolumn{9}{|l|}{ Absenteeism } \\
\hline \multicolumn{9}{|l|}{ None $(R)$} \\
\hline Yes & .053 & .018 & 2.88 & 0.006 & .022 & .018 & 1.22 & 0.230 \\
\hline \multicolumn{9}{|l|}{$\begin{array}{l}\text { Perceived adequacy } \\
\text { of staffing } \\
\text { High }(R)\end{array}$} \\
\hline Middle & .142 & .024 & 6.04 & 0.000 & .080 & .019 & 4.27 & 0.000 \\
\hline Low & 187 & .041 & 4.59 & 0.000 & .103 & .033 & 3.09 & 0.003 \\
\hline Teamwork overall scores & & & & & -.254 & .038 & -6.66 & 0.000 \\
\hline$R^{2}$ & & 0.11 & & & & 0.2 & & \\
\hline $\mathrm{F}(P)$ & & 31.14( & & & & 54.83 & & \\
\hline
\end{tabular}

Note: $(R)$ is the reference variable.

Analysis included dummy variables for study hospitals to control for their effects (output suppressed for confidentiality).

that the care will not be missed because team members believe that the team is more important than the individual staff member and that the work is "ours," not just the particular staff member's who is assigned to the patient. This leads to being aware of other team members' workload and then backing one another up by moving in to assist. Teamwork requires leadership as well, and these leadership individuals coordinate the work of the entire team, ensuring that care is not missed. Teamwork also ensures that the team adapts to the changing conditions and shares a mental model of what care is needed. These require closed-loop communication and trust. This study is limited by the use of 4 hospital sites which limits generalizability. This study utilized a survey approach to obtain information about missed nursing care and teamwork. Thus, results reflect the perceptions of the respondents as opposed to an observation of actual teamwork and missed nursing care.

\section{IMPLICATIONS}

This study provides evidence as to the importance of nursing teamwork in the inpatient setting among staff who work together to provide nursing care to patients. It points to a need to invest in methods of enhancing 
teamwork in these settings such as teamwork training, staff engagement in dealing with teamwork problems as they emerge, mentoring staff on methods of dealing with teamwork problems, and re-engineering the compensation system to reward team (versus individual) outcomes. There are also structural barriers to teamwork that need to be addressed, such as the large size of nursing teams and the lack of leadership training and development of charge nurses who are the team leaders in these settings. Studies are needed to determine the cost of these interventions in relation to the benefits (such as fewer errors, fewer lawsuits, greater staff satisfaction, and others).

This project was funded by the Blue Cross Blue Shield Foundation and the Michigan Center for Health Intervention, University of Michigan School of Nursing, National Institutes of Health, National Institute of Nursing Research (P30 NR009000).

The authors would like to acknowledge Hyunhwa Lee for her assistance with data analysis, Christopher Friese for his critical review of the manuscript and Bernadette Carroll, Courtney Andruszekiewicz, Cindy Weintraub, Deborah Mueller, and Therese Smith for their role in data collection.

\section{REFERENCES}

1. Institute of Medicine (IOM). To Err is Human: Building a Safer Health System. Washington, DC: National Academy Press; 2000.

2. Committee on the Work Environment for Nurses and Patient Safety. Keeping Patients Safe: Transforming the Work Environment of Nurses. Washington, DC: Institute of Medicine of the National Academies; 2004.

3. Kalisch B. Nurse-nurse assistant teamwork: Impact on quality and safety. Nurs Manage (In press).

4. Kalisch BJ, Landstrom G, Hinshaw AS. Missed nursing care: A concept analysis. J Adv Nurs 2009;65:1509-17.

5. Anselmi ML, Peduzzi M, Dos Santos CB. Errors in the administration of intravenous medication in Brazilian hospitals. J Clin Nurs 2007;16:1839-47.

6. Bossert E, Beecroft PC. Peripheral intravenous lock irrigation in children: Current practice. Pediatr Nurs 1994;20: 346-9.

7. Callen BL, Mahoney JE, Grieves CB, Wells TJ, Enloe M. Frequency of hallway ambulation by hospitalized older adults on medical units of an academic hospital. Geriatr Nurs 2004;25: 212-7.

8. Krishnagopalan S, Johnson EW, Low LL, Kaufman LJ. Body positioning of intensive care patients: Clinical practice versus standards. Crit Care Med 2002;30:2588-92.

9. Lee NC, Wasson DR, Anderson MA, Stone S, Gittings JA. A survey of patient education postdischarge. J Nurs Care Qual 1998;13:63-70.

10. McWhirter JP, Pennington CR. Incidence and recognition of malnutrition in hospital. BMJ 1994;308:945-8.

11. Pittet D. Compliance with hand disinfection and its impact on hospital-acquired infections. J Hosp Infect 2001;48: S40-6.

12. Pittet D, Mourouga P, Perneger TV. Compliance with handwashing in a teaching hospital. Infection Control Program. Ann Intern Med 1999;130:126-30.
13. Timmerman RA. A mobility protocol for critically ill adults. Dimens Crit Care Nurs 2007;26:175-9.

14. Kalisch BJ. Missed nursing care: A qualitative study. J Nurs Care Qual 2006;21:306-13.

15. Sochalski J. Is More Better?: The relationship between nurse staffing and the quality of nursing care in hospitals. Med Care 2004;42:67-73.

16. Schubert M, Glass TR, Clarke SP, Aiken LH, SchaffertWitvliet B, Sloane DM, et al. Rationing of nursing care and its relationship to patient outcomes: The Swiss extension of the International Hospital Outcomes Study. Int J Qual Health Care 2008;20:227-37.

17. Kalisch BJ, Landstrom G, Williams RA. Missed nursing care: Errors of omission. Nurs Outlook 2009;57:3-9.

18. Wheelan SA, Burchill CN, Tilin F. The link between teamwork and patients' outcomes in intensive care units. Am J Crit Care 2003;12:527-34.

19. Brewer BB. Relationships among teams, culture, safety, and cost outcomes. West J Nurs Res 2006;28:641-53.

20. Morey JC, Simon R, Jay GD, Wears RL, Salisbury M, Dukes KA, et al. Error reduction and performance improvement in the emergency department through formal teamwork training: Evaluation results of the MedTeams project. Health Serv Res 2002;37:1553-81.

21. Taylor CR, Hepworth JT, Buerhaus PI, Dittus R, Speroff T. Effect of crew resource management on diabetes care and patient outcomes in an inner-city primary care clinic. Qual Saf Health Care 2007;16:244-7.

22. Meterko M, Mohr DC, Young GJ. Teamwork culture and patient satisfaction in hospitals. Med Care 2004;42:492-8.

23. Cox KB. The effects of intrapersonal, intragroup, and intergroup conflict on team performance effectiveness and work satisfaction. Nurs Adm Q 2003;27:153-63.

24. Gifford BD, Zammuto RF, Goodman EA. The relationship between hospital unit culture and nurses' quality of work life. Health Care Manag 2002;47:13-25.

25. Horak BJ, Guarino JH, Knight CC, Kweder SL. Building a team on a medical floor. Health Care Manage Rev 1991;16:65-71.

26. Rafferty AM, Ball J, Aiken LH. Are teamwork and professional autonomy compatible, and do they result in improved hospital care? Qual Health Care 2001;10:32-7.

27. Kalisch BJ, Curley M, Stefanov S. An intervention to enhance nursing staff teamwork and engagement. J Nurs Adm 2007;37:77-84.

28. Kalisch BJ, Lee H. Nursing teamwork, staff characteristics, work schedules, and staffing. Health Care Manage Rev 2009;34:323-33.

29. Kalisch BJ. Nurse and nurse assistant perceptions of missed nursing care: What does it tell us about teamwork? J Nurs Adm 2009;39:485-93.

30. Salas E, Stagl K, Burke CS. 25 years of team effectiveness in organizations: Research themes and emerging needs. In: Cooper CL, Robertson IT, editors. International Review of Industrial and Organizational Psychology. New York, NY: John Wiley \& Sons; 2004. p. 47-91.

31. Kalisch BJ, Weaver SJ, Salas E. What does nursing teamwork look like? A qualitative study. J Nurs Care Qual 2009;24: 298-307.

32. Kalisch BJ, Lee H, Salas E. The development and testing of the nursing teamwork survey. Nurs Res 2010;59:42-50. 
33. Kalisch BJ, Williams RA. Development and psychometric testing of a tool to measure missed nursing care. J Nurs Adm 2009;39:211-9.

34. Sexton JB, Helmreich RL, Neilands TB, Rowan K, Vella K, Boyden J, et al. The Safety Attitudes Questionnaire: Psycho- metric properties, benchmarking data, and emerging research. BMC Health Serv Res 2006;6:44.

35. Cohen JA. power primer. Psychol Bull 1992;112:155-9.

36. Kalisch BJ, Aebersold M. Interruptions and multitasking in nursing care. Jt Comm J Qual Patient Saf.36:126-132. 